\title{
Wireless Arm Wearable Sensor Band for Long-Term Heart Rhythms Surveillance Using a Bipolar Arm-ECG Lead
}

\author{
Omar J Escalona ${ }^{1}$, Angel Villegas ${ }^{2}$, Sephorah Mukhtar $^{1}$, Gilberto Perpiñan ${ }^{3}$, David J McEneaney ${ }^{4}$ \\ ${ }^{1}$ Ulster University, Newtownabbey, United Kingdom \\ ${ }^{2}$ University of Carabobo, Valencia, Venezuela \\ ${ }^{3}$ Antonio Nariño University, Cartagena, Colombia \\ ${ }^{4}$ Craigavon Area Hospital, Portadown, United Kingdom
}

\begin{abstract}
Bipolar ECG signals obtained from sensors in a band on the left upper-arm after signal processing can provide recordings of sufficient quality for long-term ECG monitoring. We present an cable-free, wearable sensor system (WAMECG1) for bipolar arm-ECG recording and wireless data transmission over a Wi-Fi link. The system's functional blocks were integrated into an ergonomically designed armband ECG device.

A retrospective pilot analysis of the WASTCArD armECG mapping database from our previous work, was perforned to obtain the optimal axis rotation of the bipolar electrodes pair with respect to the frontal ECG plane and the arm axilla point. The optimal signal was found to be at $-30^{\circ}$ axis rotation. Then, signal quality of the recorded farfield bipolar arm-ECG was validated in a pilot trial with 10 volunteer subjects at rest using the prototype device. The overall $R$ peak detection accuracy was $99.67 \%$. Without using any signal enhancement algorithm, the average signal-to-noise-ratio (SNR) values was 16.73. These performance assessment results validated the performance of the wearable arm-band prototype device.
\end{abstract}

\section{Introduction}

Cardiovascular disease (CVD), is the commonest cause of death in the world, accounting for an estimated $31.5 \%$ of all global deaths [1]. In the United States, CVD claims more lives annually than cancer and chronic lung disease combined [2]. Sudden cardiac death (SCD), usually caused by lethal arrhythmias, accounts for $44.13 \%$ of total CVD deaths [2]. Early arrhythmia detection reduces mortality from SCD. Most cardiac arrhythmias are initially sporadic, lasting only a few seconds. Long-term cardiac monitoring would permit early detection of transient arrhythmias. Currently available technologies for screening for cardiac arrhythmias include implantable loop recorders (ILR) for long-term and Holter monitors for shorter intervals. These approaches have their advantages and drawbacks as discussed in [3].

Recent advances in embedded wireless communication and sensor technologies have led to increased application of these devices in healthcare [4]. It is expected that new cost-effective, non-invasive, long-term ECG monitoring alternatives would reduce mortality in subjects at high risk for SCD [5].

Modern ECG monitoring devices based on various wireless technologies including Bluetooth, ZigBee or WiFi have been developed recently [6]. Wi-Fi based systems offer higher data transmission rate than other wireless communication interfaces available in most smartphones, laptops or personal computers but have the drawback of being less power efficient.

Experimental wearable ECG devices currently under development include T-shirts, vests, belts, patches and armbands [7]. In this context, armband devices are convenient for long-time recording on the upper arm and previous research carried by our team [8] confirmed the feasibility of denoising, processing, analysing, and extracting information from low-amplitude far-field ECG potentials [9].

We here present a novel upper-arm wearable band prototype device for arm-ECG signal acquisition. In addition, we analyse the optimal positioning of the wearable device on the left upper arm.

\section{Methods}

\subsection{Optimal bipolar arm-lead location}

The WASTCArD arm-ECG mapping database of 10 cases created in a previous study [5] was used to find the best deployment of the electrode pair around the upper left arm with reference to the frontal body plane. Six possible bipolar leads, derived from the five upper left-arm unipolar channels (referenced to ground (GND)) as shown in Figure 1 generate angles, corresponding to electrodes axis rotations around the axilla, as noted in Table 1 . The bipolar Lead-1 $[\mathrm{Ch} 10-\{(\mathrm{Ch} 8+\mathrm{Ch} 9)\} / 2]$, gelled electrodes and Lead-6 (Ch13-Ch14) dry electrode, both represent the baseline axis with $0^{\circ}$ rotation. However, the axes of bipolar leads generated from $\mathrm{Ch} 8$ and $\mathrm{Ch} 9$ with respect to $\mathrm{Ch} 10$ (gelled electrodes) is not $0^{\circ}$, as may be perceived from 
Figure 1; where, the axes of Lead-2 $\left(30^{\circ}\right)$ and Lead-5 ( $-30^{\circ}$ or $330^{\circ}$ ) form about equal but opposite angles. Lead-3 $\left(300^{\circ}\right.$ or $\left.-60^{\circ}\right)$ and lead $-4\left(90^{\circ}\right)$ depict distal (from Ch10) near and far axis respectively. Nevertheless, the rotation angles may vary slightly, due to the fixed sensor strap length (BIS-Quatro ${ }^{\mathrm{TM}}$ ) and the actual subject's am diameter.

The signal-to-noise ratio (SNR) of the bipolar leads was analysed to determine the best bipolar arm lead location; high SNR corresponding to ECG signal quality for optimal lead rotational positioning.
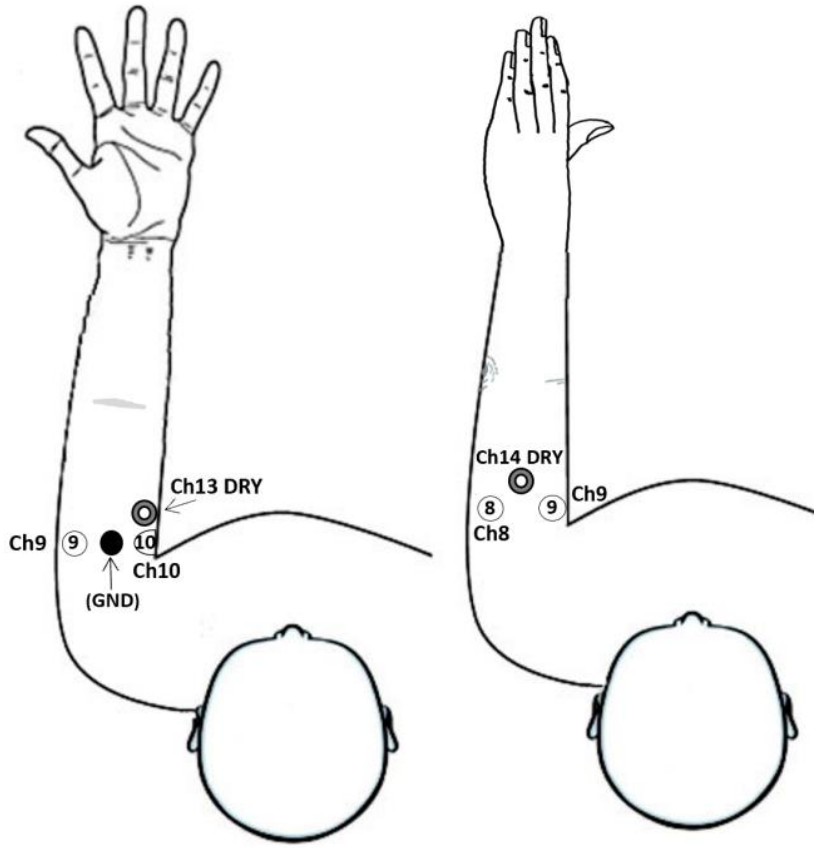

Figure 1. Placement of bipolar-leads on the upper left arm

A 20 second ECG recording, at a sampling rate of 2048 $\mathrm{Hz}$ was extracted from each of the 10 cases in the WASTCArD upper-arm ECG mapping database, and was digitally filtered, using a $50 \mathrm{~Hz}$ notch filter with a Q-factor of 20. Pre-processing was implemented using bandpass filtering process which included a $2^{\text {nd }}$ order Butterworth high-pass filter $\left(0.7 \mathrm{~Hz}\right.$ cutoff frequency) followed by a $4^{\text {th }}$ order low-pass filter ( $40 \mathrm{~Hz}$ cutoff frequency) to reduce unwanted noise and obtain a clear signal.

Next, the positions of the QRS complexes in these bipolar arm leads in each of the 10 cases were annotated. A $160 \mathrm{~ms}$ signal window centred around the $\mathrm{R}$ peak and a $40 \mathrm{~ms}$ ECG baseline noise window (see Figure 2) were used to calculate the amplitude standard deviation (SD) of QRS signal and noise and obtain the signal-to-noise ratio (SNR) for each arm-lead in all cases for this pilot study. The average value of the SNRs in the 10 cases, after excluding the SNR outliers for each analysed bipolar arm-lead position are presented in Table1.

The SNR analysis showed bipolar Lead-5 (Ch10-Ch8) to be the best position (at an inter-electrode axis rotation of $-30^{\circ}$ ) thus providing a guide for positioning the arm band strap (see Figure 4).

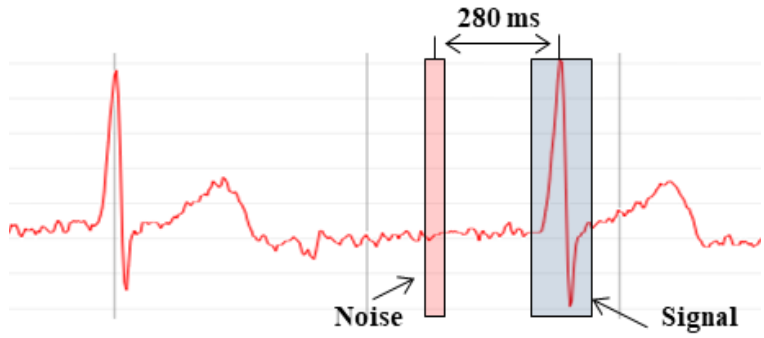

Figure 2. Signal and noise measurement windows for SNR calculation on arm-ECG signals.

Table 1. Analysis of bipolar arm-lead location: Average SNR [SD of signal $(160 \mathrm{~ms}) / \mathrm{SD}$ of noise $(40 \mathrm{~ms})$ ] of six bipolar leads at different axis rotation angles.

\begin{tabular}{lcc}
\hline $\begin{array}{l}\text { Bipolar Arm-Lead Name } \\
\text { and Definition }\end{array}$ & $\begin{array}{c}\text { Bipolar Arm-Lead Axis Relative } \\
\text { Rotation Angle and Position }\end{array}$ & $\begin{array}{c}\text { S/N Average } \\
\text { Value }(\mathbf{N}=\mathbf{1 0})\end{array}$ \\
\hline $\begin{array}{lcc}\text { Lead-1: } \\
\text { [Ch10- }\{(\mathrm{Ch} 8+\mathrm{Ch} 9)\} / 2]\end{array}$ & $\mathbf{0}^{\circ}$ (Gelled electrodes $)$ & $\mathbf{1 2 . 1 7}$ \\
Lead-2: $[\mathrm{Ch} 10-\mathrm{Ch} 9]$ & $\mathbf{3 0}^{\circ}$ & $\mathbf{9 . 0 1}$ \\
Lead-3: $[\mathrm{Ch} 8-\mathrm{GND}]$ & $\mathbf{3 0 0}^{\circ}\left(\right.$ or $^{\left.-\mathbf{6 0}^{\circ}, \text { Distal, near axis }\right)}$ & $\mathbf{1 5 . 3 0}$ \\
Lead-4: $[\mathrm{Ch} 8-\mathrm{Ch} 9]$ & $\mathbf{9 0}^{\circ}($ Distal, far axis $)$ & $\mathbf{1 1 . 6 0}$ \\
Lead-5: $[\mathrm{Ch} 10-\mathrm{Ch} 8]$ & $\mathbf{3 3 0}^{\circ}\left(\right.$ or $\left.-\mathbf{3 0}^{\circ}\right)$ & $\mathbf{1 5 . 8 0}$ \\
Lead-6: $[\mathrm{Ch} 13-\mathrm{Ch} 14]$ & $\mathbf{0}^{\circ}$ (Dry electrodes $)$ & $\mathbf{1 3 . 1 9}$ \\
\hline
\end{tabular}

\subsection{Sensor system description}

The system consisted of two components: a wearable arm-band smart sensor (WAMECG1) and a Web client application (ECGView). The HTTP protocol was used to exchange messages between a server embedded in the WAMECG1 and a client computer or smartphone running the application for monitoring and configuration. After the system is configured and signal acquisition commenced up to 72 hours recording can be stored to the internal nonvolatile memory. Afterwards, the Wi-Fi connection is no longer required until the data is downloaded for further analysis. The architecture depicted in Figure 3.



Figure 3. System architecture. 


\subsection{Hardware design}

The WAMECG1 sensor was designed around the ESP8266 system on a chip which includes a high performance 32-bit processor and $802.11 / \mathrm{b} / \mathrm{g} / \mathrm{n}$ radio transceiver for wireless communication in a reduced-size package. A single PCB contains the main processing unit with the analogue front-end circuitry for signal conditioning, acquisition and storage. The bipolar armECG acquired data is stored in a microSD memory card in a binary file using the European Data File format (EDF) widely used in biological signal acquisition systems.

The arm-ECG signal conditioning chain was designed using the AD8232 analogue front end. This integrated circuit is a monolithic solution for ECG and other bipotential measurements applications. Using additional external components, the AD8232 was configured accordingly to the arm-ECG acquisition requirements. A two-pole high pass circuit with cut-off frequency of $0.7 \mathrm{~Hz}$ was used to reduce low frequency motion artefact (baseline wandering), while a two-pole high pass filter with cut-off frequency of $40 \mathrm{~Hz}$ attenuated the higher frequency electromyopotential noise. A step-down resistor network with an additional one-pole RC anti-aliasing filter was used to interface the AD8232 to the ESP8266 microcontroller. Power line interference was mitigated using a right-leg drive circuit and incorporating a 2-pole digital IIR filter implemented microcontroller for the acquisition firmware. The differential input range of sensor was defined in $\pm 1500 \mu \mathrm{V}$ offering a total resolution of $2.93 \mu \mathrm{V}$.

\subsection{Software architecture}

A three-layer architecture was used for the embedded firmware and ECGView application. At the firmware level, the Hardware layer contained all drivers required to manipulate the internal hardware resources of the ESP8266 $\mathrm{SoC}$ and interface the external peripherals of the system; it communicates with the domain layer which contains the ECG acquisition and local processing sub-layers and a shared memory space where both sub-layers perform their read and write operations. A control module handles the access to shared memory. The Application Layer controls the overall firmware operation and processes the HTTP requests.

The client-side software of the system is a Web application called ECGView, developed to configure and control the operation of WAMECG1 sensor. It was created in JavaScript language and runs in a standard web browser. The application displays a graphical interface where the user can select the operation mode allowing visualisation of the arm-ECG signal in real-time or loaded data from a previously recorded ECG file. Using the on-screen controls, the user can configure the acquisition parameters (sampling rate, duration of the recording and notch filter
Q-factor), adjust the arm-ECG graph appearance (adjusting zoom, pan or scroll the trace for visual inspection), start/stop the data acquisition or recall the armECG data stored in the sensor.

\subsection{Performance metrics for arm-ECG signal quality assessment}

Signal-to-noise ratio (SNR), as defined in Figure 2, was selected as the metric for evaluating the quality of the armECG signals acquired by the WAMECG1 prototype device. R-peak counting was incorporated as an additional performance indicator of the acquired signals.

\section{Results}

The completely assembled system is shown in Figure 4. All the electronic components including a rechargeable LiPo battery were fitted in an ergonomic PVC case. The reference and negative electrodes are placed in the enclosure whereas the third electrode is located on the elastic band. Table 2 summarizes the specifications achieved by the WAMECG1 prototype device.

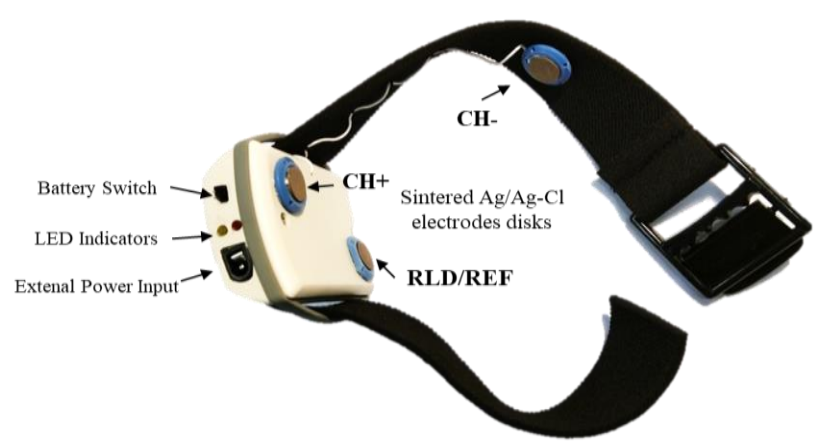

Figure 4. WAMECG1 sensor fully assembled.

Table 2. WAMECG1 System specifications.

\begin{tabular}{cc}
\hline Item & Value \\
\hline Sampling rate & 125,250,500 Hz \\
Data transmission & Wi-Fi 2.5 Mbps avg. transfer \\
rate & MicroSD card (up to 32GB) \\
Storage media & Up to 72hours \\
Operation time & $18 \mathrm{~mA}$ (normal operation) \\
& $36 \mathrm{~mA}$ (real-time mode) \\
Average power & $\sim 100 \mathrm{~mA}$ (file transmission) \\
consumption & $5 \mathrm{VDC}$ to 7.5VDC \\
Power input &
\end{tabular}

\subsection{Initial System testing and calibration}

The WAMECG1 sensor output was digitally calibrated against a high-resolution $(0.305 \mathrm{uV})$ medical-grade ECG acquisition system model ECG-1200HR (Norav Medical Delray Beach, FL, USA). A synthetic normal sinus rhythm 
(NSR) signal was simultaneously applied to both WAMECG1 and the reference unit as seen in Figure 5a. The recorded output was used to calculate the offset and gain correction factors for our sensor. After applying a second-order, zero-phase $0.7 \mathrm{~Hz}$ to $40 \mathrm{~Hz}$ band-pass filter on the signals from both systems they were visually indistinguishable as shown in Figure 5b-5c. The calculated root-mean-square error (RMSE) was $11.08 \mathrm{uV}$ (corresponding to $0.34 \%$ of the WAMEGC1 full scale) and the cross-correlation coefficient (CC) with the reference system was 0.997.

(a)

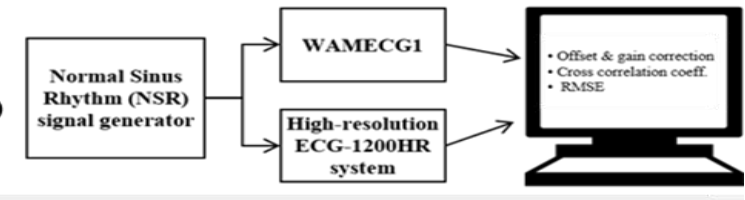

(b)

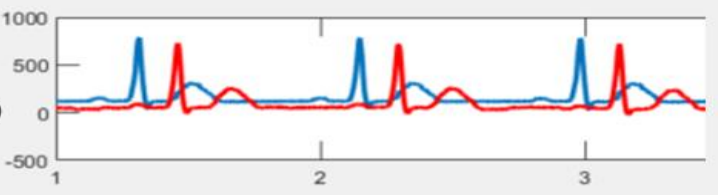

(c)

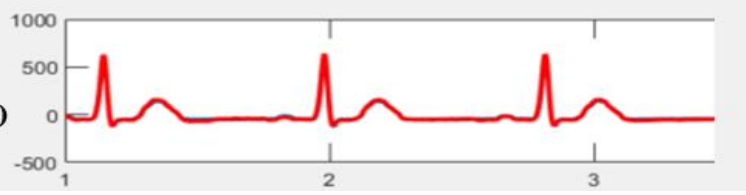

Figure 5. Offset and amplitude calibration: (a) Experimental setup, (b) acquired un-calibrated signals, (c) overlapping signals after calibration and filtering.

\subsection{Experimental validation}

Ten male participants recruited from the research group (average age 39.1+SD15.1 years old) agreed to participate in the WAMECG1 validation test. The arm-ECG sensor band was placed on the left arm. SNR was calculated for all subjects (see Table 3). Participants S3 and S6 exhibited the highest (30.32) and lowest (8.45) SNR values respectively. The average SNR for all the participants was 16.73 (SD 6.22). The average SNR in the best 5 cases was 21.43 (SD 4.65). The overall accuracy in the detection of R peaks was $99.67 \%$ (611 of 613 correctly detected).

Table 3. SNR values for 10 participants

\begin{tabular}{cccccccccccc}
\hline & P1 & P2 & P3 & P4 & P5 & P6 & P7 & P8 & P9 & P10 $\begin{array}{c}\text { Mean } \\
\text { SNR }\end{array}$ \\
\hline S/N & 17.1 & 21.7 & 30.3 & 18.5 & 14.9 & 8.4 & 19.2 & 9.3 & 10.3 & 17.5 & 16.7
\end{tabular}

\section{Discussion and Conclusion}

An arm-ECG band was constructed and validated as an innovative sensor system solution for the acquisition of high quality bipolar far-field ECG signals on the left upper arm. The system offers high levels of user comfort compared to traditional wired sensing systems and is capable of stand-alone operation.

The optimal positioning analysis of bipolar electrodes pair in the WAMECG1 sensor arm-band, was estimated to be at axis relative rotation of $-30^{\circ}$. This confirmed the initial findings from our previous work $[6,8]$. A limitation in this pilot analysis was the small database with only 10 subjects. An ongoing study with a larger clinical database, including more than 120 subjects, will provide a more precise analysis for the best position of the arm-band electrodes.

The arm-ECG QRS complex position was accurately detected on $99.67 \%$ of the recorded heartbeats confirming the capability of monitoring variations of the heart rate using this system.

Another observed limitation of the current system is the battery autonomy. Optimisation of power consumption will be required while the data acquisition is in progress.

\section{References}

[1] Roth, G.A., et al.. "Global and Regional Patterns in Cardiovascular Mortality From 1990 to 2013. Circulation, 132:1667-1678, 2015.

[2] Virani, S.S., et al., "Heart Disease and Stroke Statistics 2020 Update: A Report from the American Heart Association (2020)", Circulation, 141: e139-e596, 2020.

[3] Lynn, W.D., Escalona, O J., McEneaney, D.J. "Arm and Wrist Surface Potential Mapping for Wearable ECG Rhythm Recording Devices: A Pilot Clinical Study". Journal of Physics: Conference Series, 450, 2013.

[4] Dey, N.; Ashour, A.S.; Shi, F. et al. Developing Residential Wireless Sensor Networks for ECG Healthcare Monitoring. IEEE Trans. Consumer Electronics, 63(4): 442-449, 2017.

[5] Escalona, O, McFrederick, L, Borge, M, Perpiñan, G, McLaughlin, J, McEneaney, D. "Wrist and Arm Body Surface Cardiac Electrogram Mappping Techniques Study for Long-Term Rhythm Monitoring". In Computing in Cardiology; 44, 2017.

[6] Villegas, A, McEneaney, D, Escalona, O. “Arm-ECG Wireless Sensor System for Wearable Long-Term Surveillance of Heart Arrhythmias". Electronics, vol.8, no.11, 1300,2019.

[7] Lynn, WD, Escalona, OJ, et al. "Arm-ECG Bipolar Leads Signal Recovery Methods for Wearable Long-Term Heart Rate and Rhythm Monitoring”. Comp. in Cardiol., 44, 2017.

[8] Escalona, O., Lynn, W., Perpiñan, G., et al. "Data-Driven ECG Denoising Techniques for Characterising Bipolar Lead Sets Along the Left Arm in Wearable Long-Term Heart Rhythm Monitoring”, Electronics, vol. 6, no. 4, p. 84, 2017.

[9] Vizcaya P., Perpiñan G., McEneaney D., Escalona O.J. "Standard ECG Lead I Prospective Estimation Study from Far-Field Bipolar Leads on the Left Upper Arm: A Neural Network Approach". J. Biomed. Signal Process. \& Control, 51, 171-180.

Address for correspondence:

Name: Omar J Escalona

Full postal address: Ulster University, Shore Road,

Newtownabbey, BT37 0QB, United Kingdom.

E-mail address: oj.escalona@ulster.ac.uk 